\title{
Robust de novo pathway enrichment with KeyPathwayMiner 5
}

\section{[version 1; peer review: 2 approved]}

\section{Nicolas Alcaraz'1,2, Markus List'-5, Martin Dissing-Hansen1", Marc Rehmsmeier6, Qihua Tan4,7, Jan Mollenhauer2,3, Henrik J. Ditzel2,3,8, Jan Baumbach1,5}

\author{
${ }^{1}$ Department of Mathematics and Computer Science, University of Southern Denmark, 5230 Odense, Denmark \\ ${ }^{2}$ Department of Cancer and Inflammation Research, Institute of Molecular Medicine, University of Southern Denmark, 5000 Odense, \\ Denmark \\ ${ }^{3}$ Lundbeckfonden Center of Excellence in Nanomedicine NanoCAN, University of Southern Denmark, 5000 Odense, Denmark \\ ${ }^{4}$ Institute of Clinical Research, University of Southern Denmark, 5000 Odense, Denmark \\ ${ }^{5}$ Max Planck Institute for Informatics, 66123 Saarbrucken, Germany \\ ${ }^{6}$ Integrated Research Institute (IRI) for the Life Sciences and Department of Biology, Humboldt-Universitat zu Berlin, 10099 Berlin, \\ Germany \\ 7Epidemiology, Biostatistics and Biodemography, Institute of Public Health, University of Southern Denmark, 5000 Odense, \\ Denmark \\ ${ }^{8}$ Department of Oncology, Odense University Hospital, 5000 Odense, Denmark
}

V1 First published: 28 Jun 2016, 5:1531

https://doi.org/10.12688/f1000research.9054.1

Latest published: 28 Jun 2016, 5:1531

https://doi.org/10.12688/f1000research.9054.1

\section{Abstract}

Identifying functional modules or novel active pathways, recently termed de novo pathway enrichment, is a computational systems biology challenge that has gained much attention during the last decade. Given a large biological interaction network, KeyPathwayMiner extracts connected subnetworks that are enriched for differentially active entities from a series of molecular profiles encoded as binary indicator matrices. Since interaction networks constantly evolve, an important question is how robust the extracted results are when the network is modified. We enable users to study this effect through several network perturbation techniques and over a range of perturbation degrees. In addition, users may now provide a gold-standard set to determine how enriched extracted pathways are with relevant genes compared to randomized versions of the original network.

Keywords

Pathway enrichment, network analysis, data integration, algorithms, systems biology

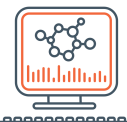

This article is included in the Bioinformatics

gateway.

\section{Open Peer Review}

\begin{tabular}{lcc} 
Approval Status & 1 \\
\hline version 1 & 1 & 2 \\
28 Jun 2016 & view & view
\end{tabular}

1. Alberto Calderone (D), Molecular Genetics Laboratory - Tor Vergata University, Rome, Italy

2. Mona Riemenschneider, Weihenstephan-

Triesdorf University of Applied Science,

Straubing, Germany

Any reports and responses or comments on the article can be found at the end of the article. 


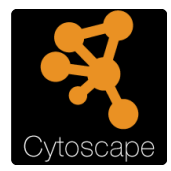

This article is included in the Cytoscape

gateway.

This article is included in the Max Planck Society

collection.

Corresponding author:Jan Baumbach (jbaumbac@imada.sdu.dk)

Competing interests: No competing interests were disclosed.

Grant information: This work was supported by the Lundbeckfonden grant for the NanoCAN Center of Excellence in Nanomedicine, the Region Syddanmarks ph.d.-pulje and Forskningspulje, the Fonden Til Lægevidenskabens Fremme, by the DAWN-2020 project financed by Rektorspuljen SDU2020 program, the MIO project of the OUH Frontlinjepuljen, the Bioinformatics part of NEXT - National Experimental Therapy Partnership funded by the Innovation Fund Denmark, as well as the VILLUM foundation by a Blokstipendiet. NA would like to acknowledge el Consejo Nacional de Ciencia y Tecnología (CONACyT) from Mexico for their financial support. The funders had no role in study design, data collection and analysis, decision to publish, or preparation of the manuscript.

Copyright: ( 2016 Alcaraz $\mathrm{N}$ et al. This is an open access article distributed under the terms of the Creative Commons Attribution License, which permits unrestricted use, distribution, and reproduction in any medium, provided the original work is properly cited. Data associated with the article are available under the terms of the Creative Commons Zero "No rights reserved" data waiver (CC0 1.0 Public domain dedication).

How to cite this article: Alcaraz N, List M, Dissing-Hansen M et al. Robust de novo pathway enrichment with KeyPathwayMiner 5 [version 1; peer review: 2 approved] F1000Research 2016, 5:1531 https://doi.org/10.12688/f1000research.9054.1

First published: 28 Jun 2016, 5:1531 https://doi.org/10.12688/f1000research.9054.1 


\section{Introduction}

De novo pathway enrichment methods have gained much attention during the last decade due to their potential to identify novel regulators and putative biomarkers from vast datasets in systems biology research. Given a biological interaction network, such as defined by BioGrid ${ }^{1}$, IntAct ${ }^{2}$ or $\mathrm{I}^{2} \mathrm{D}^{3}$, the main objective of de novo pathway enrichment is to extract connected subnetworks that are enriched for genes that are implicated in the phenotype of interest. This phenotype is dependent on the experiment and observed in one or several omics datasets, including, for instance, gene expression values, DNA methylation signals or single nucleotide variants. The common denominator of de novo pathway enrichment methods is that the resulting subnetworks are expected to include known pathways as well as novel pathways that have little overlap with annotated pathways found in curated databases. Existing approaches can be divided into the following categories: (i) aggregate score optimization methods, where the objective is to extract subnetworks that maximize a summary or statistical score of the individual gene scores, (ii) score propagation methods, where individual gene scores from the molecular profiles are propagated through the network, or adjusted to reflect also their connectivity in the network, (iii) module cover approaches, where the objective is to extract subnetworks containing genes that cover as many active cases/samples as possible, and (iv) cluster-based approaches. Methods that fall into categories (i), (ii) or (iv) rely heavily on the scoring function, which must be appropriate for the technology of the molecular profile being studied. In contrast, methods based on the module cover approach (iii) do not suffer from this issue, but leave it up to the user to find a sensible way to discern active from inactive genes. An overview of popular de novo pathway enrichment methods is shown in Table 1 . We identify three issues common to existing de novo methods:

- There is little consensus on what constitutes a novel pathway. It is up to the user to find method-specific parameters that lead to a satisfying solution. Choosing these parameters is often not intuitive and even small changes can lead to large variations in the results. Most methods provide little guidance on parameter selection, forcing users to rely on educated guesses, or to tediously re-run the method multiple times until the optimal parameters for a given analysis are found.

- It has been demonstrated that for several methods results change significantly upon perturbations in the underlying networks ${ }^{4}$. This lack of robustness is an issue, since interaction databases are continuously evolving and it is unclear to what degree the results will change when a particular tool is applied with the exact same data to a newer version of the network.

- In the rare cases where a ground truth or gold standard is available, the validation of de novo pathway enrichment results is not straightforward and, to our knowledge, not supported by any available method.

We have previously developed KeyPathwayMiner, a de novo pathway enrichment tool following the module cover approach. Even though the parameters in KeyPathwayMiner are relatively intuitive, their selection becomes challenging for analyses involving several distinct omics datasets. To address this issue, we allow users to define a range (consisting of minimum, maximum and step size) for each parameter. The resulting grid search is fully automated and saves the user from going through tedious repetitions. While testing different parameters is more convenient in this way, it is still necessary to manually inspect the resulting subnetworks to select the optimal settings in a subjective fashion.

Here, we present version 5 of KeyPathwayMiner, which is the first tool to provide a user-friendly way to systematically evaluate the quality and robustness of the results in de novo pathway enrichment. We achieve this by perturbing the input network to

Table 1. A non-exhaustive selection of popular de novo network

enrichment tools. Abbreviations: Cytoscape app (CA), standalone version/ package (SA), desktop application (DA), web application (WA), web service (WS), visualization (VIZ), multi-omics (MO), robustness of the results upon network perturbation (RB), validation of the results using a gold standard upon network perturbation (VL)

\begin{tabular}{|l|c|c|c|c|c|c|c|c|c|}
\hline & CA & SA & DA & WA & WS & VIZ & MO & RB & VL \\
\hline BioNET $^{5}$ & $x$ & $\checkmark$ & $x$ & $x$ & $x$ & $\checkmark$ & $x$ & $x$ & $x$ \\
\hline GiGa $^{6}$ & $x$ & $\checkmark$ & $x$ & $x$ & $x$ & $x$ & $x$ & $x$ & $x$ \\
\hline GXNA $^{7}$ & $x$ & $\checkmark$ & $x$ & $x$ & $x$ & $x$ & $x$ & $x$ & $x$ \\
\hline HotNet $^{8}$ & $x$ & $\checkmark$ & $x$ & $x$ & $x$ & $\checkmark$ & $x$ & $x$ & $x$ \\
\hline jActiveModules $^{9}$ & $\checkmark$ & $x$ & $x$ & $x$ & $x$ & $\checkmark$ & $x$ & $x$ & $x$ \\
\hline MATISSE $^{10}$ & $x$ & $x$ & $\checkmark$ & $x$ & $x$ & $\checkmark$ & $x$ & $x$ & $x$ \\
\hline PinnacleZ $^{11}$ & $\checkmark$ & $x$ & $x$ & $x$ & $x$ & $\checkmark$ & $x$ & $x$ & $x$ \\
\hline RegMOD $^{12}$ & $x$ & $\checkmark$ & $x$ & $x$ & $x$ & $x$ & $x$ & $x$ & $x$ \\
\hline KPM 5.0 $^{2}$ & $\checkmark$ & $\checkmark$ & $x$ & $\checkmark$ & $\checkmark$ & $\checkmark$ & $\checkmark$ & $\checkmark$ & $\checkmark$ \\
\hline
\end{tabular}


varying degrees. Depending on the research question, several perturbation techniques are available. To assess robustness of the results, the largest solution found in the perturbed network(s) is compared against the largest solution found in the unperturbed network. The size and variance of the overlap is illustrated for different user-controlled levels of perturbation and is an indicator for the robustness of the results. If a gold standard is available, an additional measure of quality is the overlap of the largest solution found in the unperturbed as well as in the perturbed network(s) with the gold standard. As an example application case, we apply KeyPathwayMiner to a gene expression dataset covering 38 Huntington's disease patients and 32 healthy controls. We demonstrate the potential of network perturbation to help assessing the quality and robustness of the extracted results.

\section{Methods}

Implementation

KeyPathwayMiner is implemented as a modular Java application centering on a core module that provides various de novo pathway enrichment strategies and methods for network perturbation analysis and plotting. A number of application modules have been implemented for different usage scenarios, including a standalone module, a web application module ${ }^{13}$, and a Cytoscape app module. The web application module KeyPathwayMinerWeb (http://keypathwayminer.compbio.sdu.dk), for instance, is primarily targeted at researchers with little to no experience in Cytoscape. No installation is necessary and convenience features, such as the mapping of identifiers or the conversion of $\mathrm{p}$-value matrices to indicator matrices, are included. Web application developers may utilize a RESTful interface to integrate KeyPathwayMinerWeb seamlessly into their own applications. The standalone version is targeted at developers and data analysts who need more computational power than KeyPathwayMinerWeb offers and thus seek to incorporate KeyPathwayMiner directly in their own software implementation or in data analysis scripts, respectively. Finally, the Cytoscape app is the most powerful module, since it is also not limited with respect to the parameter range and computational power needed but also offers additional useful features such as combining OMICs datasets with a logical formula editor or the generation of plots using the JFreeChart (www.jfreechart.org) library.

\section{Operation}

After installing the KeyPathwayMiner Cytoscape app via the app store, the user is expected to load an interaction network into Cytoscape. The KeyPathwayMiner tab can be found in the Control Panel and allows for one or more indicator matrices to be selected as input under the initial tab 'Data'. These matrices can be derived from OMICs datasets such that samples correspond to columns and nodes (genes) to rows. Each entry in the matrix is either a ' 1 ' indicating an active case in a node or ' 0 ' otherwise. A typical example is a gene expression dataset in which a ' 1 ' represents a differentially expressed gene. Another example could be a nextgeneration sequencing dataset where a ' 1 ' indicates a single nucleotide polymorphism. Example files can be downloaded from the KeyPathwayMiner website at http://keypathwayminer.compbio. sdu.dk (Figure 1A). In the next tab, called 'Links', the user can customize how several datasets are combined for the analysis. Here, one can choose between 'AND' (a case is considered active if it is active in all datasets), 'OR' (a case is considered active if it is active in any of the datasets), or 'CUSTOM', which allows for connecting datasets in an interactive formula editor (Figure 1B). The tab 'Pos/Neg' allows the user to define nodes that are always considered active (positive list) or that are ignored (negative list). In the 'Run' tab, it is finally possible to select the parameters for the KeyPathwayMiner run. Batch runs can also be performed by defining a range of values for $K$ and $L$, such that users can conveniently run and assess the results for varying values of these parameters. (Figure 1C). KeyPathwayMiner relies on two easy-to-interpret parameters to control the size of the extracted subnetworks. The user can choose between a local as well as a global enrichment strategy. In the local strategy, INEs (Individual Node ExceptionS), a gene is considered active when it is active in all but $L$ cases/ samples. In addition, a parameter $K$ allows KeyPathwayMiner to add additional inactive genes to extend the size of the solutions. We observe that INES has a tendency to prefer hub nodes, which is not always desirable. We therefore implemented the GLONE (GLobal Node Exceptions) strategy, where the parameter $L$ is considered across all genes and fewer hub nodes are selected at the cost of run-time.

The optimal values for $K$ and $L$ depend on the datase ${ }^{14}$. KeyPathwayMiner allows users to define a range for both parameters to identify the best settings in a straight-forward fashion.

Users can choose between different methods to extract subnetworks: an exact (fixed parameter tractable), a greedy, and a heuristic (ant colony optimization) algorithm. For additional details regarding KeyPathwayMiner we refer to ${ }^{14-16}$.

Several new features have been implemented in version 5 of KeyPathwayMiner and are described in the following.

\section{Network perturbation}

KeyPathwayMiner now enables users to study the robustness and validity of the extracted subnetworks through perturbation (Figure 1D). To this end, the user can choose from the following common strategies:

- Node label permutation: Pairs of nodes are selected arbitrarily and their node labels are swapped. This technique preserves the network structure exactly, but affects the local density of active genes in the network.

- Degree preserving rewiring: In this strategy first suggested by Maslov et al. ${ }^{17}$, two arbitrary edges are selected and their endpoints are swapped. As a result, the local network structure is actively changed while the global topological structure and the node degree distribution remain intact. With a large number of permutations this strategy leads to a randomized network.

- Node removal: In this strategy, a certain percentage of arbitrarily selected nodes are removed, thus simulating what results on a less complete network would look like. This is particularly interesting since interaction networks are continuously growing in size.

- Edge removal: In contrast to node removal, which affects network size, this strategy affects primarily the density of a network. 


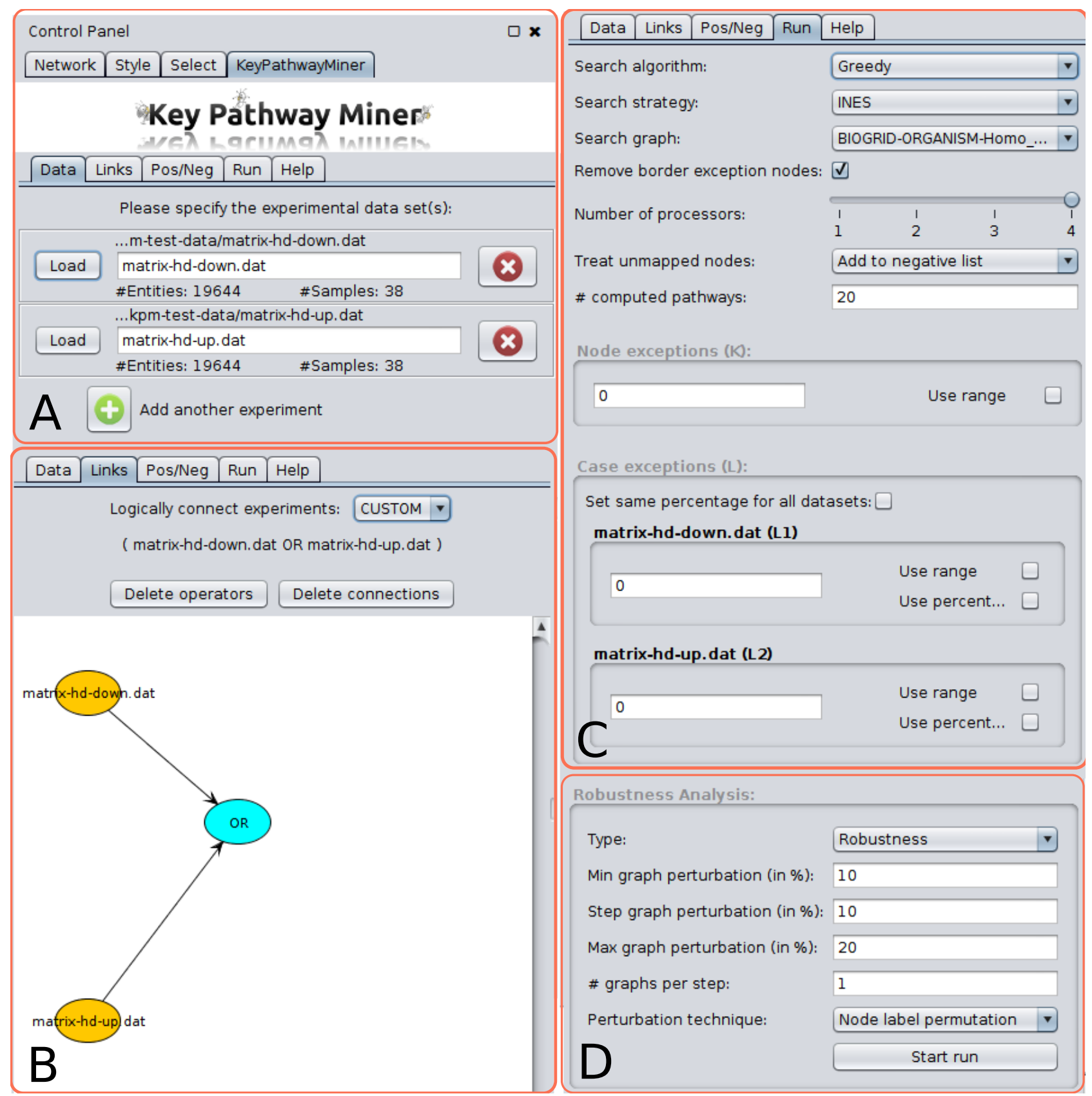

Figure 1. The user interface of the KeyPathwayMiner Cytoscape app is located in the control panel. The user sets the analysis up as follows (omitting the 'Pos/Neg' tab, where nodes can be specified for inclusion or exclusion): (A) one or several dataset files are selected from the disk. (B) Several dataset files can be logically connected via a formula editor. (C) The run parameters are configured, most importantly the enrichment strategy (INES or GLONE), the search algorithm, the input network and the search parameters $K$ and/or $L$, which can also be defined as a range. (D) Network perturbation settings used in robustness or validation runs. 


\section{Robustness analysis}

To assess the quality of the results, we consider the overlap of the largest solution between the various perturbed and the nonperturbed analyses. With an increasing degree of perturbation of the network, it can be expected that this overlap will decrease. Users can thus assess how robust the observed result is by considering the Jaccard similarity coefficient between the gene sets $S$ and $S_{\text {unperturbed }}$ based on the gene sets extracted from the largest solution found using the perturbed and non-perturbed networks, respectively:

$$
J\left(S_{\text {perturbed }}, S_{\text {unperturbed }}\right)=\frac{\left|S_{\text {perturbed }} \cap S_{\text {unperturbed }}\right|}{\left|S_{\text {perturbed }} \cup S_{\text {unperturbed }}\right|}
$$

Validation analysis

Similarly, the comparison of the overlap of the largest solution of the perturbed as well as the non-perturbed analyses and a gold standard $S_{\text {Gold }}$ can be used as a quality metric:

$$
\begin{gathered}
J\left(S_{\text {perturbed }}, S_{\text {gold }}\right)=\frac{\left|S_{\text {perturbed }} \cap S_{\text {gold }}\right|}{\left|S_{\text {perturbed }} \cup S_{\text {gold }}\right|} \\
J\left(S_{\text {unperturbed }}, S_{\text {gold }}\right)=\frac{\left|S_{\text {unperturbed }} \cap S_{\text {gold }}\right|}{\left|S_{\text {unperturbed }} \cup S_{\text {gold }}\right|}
\end{gathered}
$$

\section{New $L$ parameter specification options}

As a convenience feature, we now allow users to select $L$, which allows users to define the number of case exceptions allowed in a solution, to be defined as a percentage of the total number of cases. This is particularly advantageous in the case of multiple datasets, where the $L$ parameter (range) can now be selected once for all datasets in spite of differences in the number of cases between them.

\section{Border exception node removal}

The INES model extracts subnetworks with up to $K$ exception nodes that are not active or differentially expressed (as defined by the $L$ parameter). In many cases, these exception nodes are central in the pathway, i.e. they connect (groups of) active genes.
However, if the $K$ parameter is too large, some of these exception nodes are simply added to the periphery of the subnetwork to increase the size of the solution (Figure 2). As a result, the top solutions of a KeyPathwayMiner run would sometimes consist of overlapping subnetworks that only differ in these border exception nodes (BENs). BENs can now be removed in an optional filtering step, which will lead to more diverse solutions.

BENs are removed as shown in Algorithm 1, which has worstcase running time of $O((|V|+|E|) * K)$.

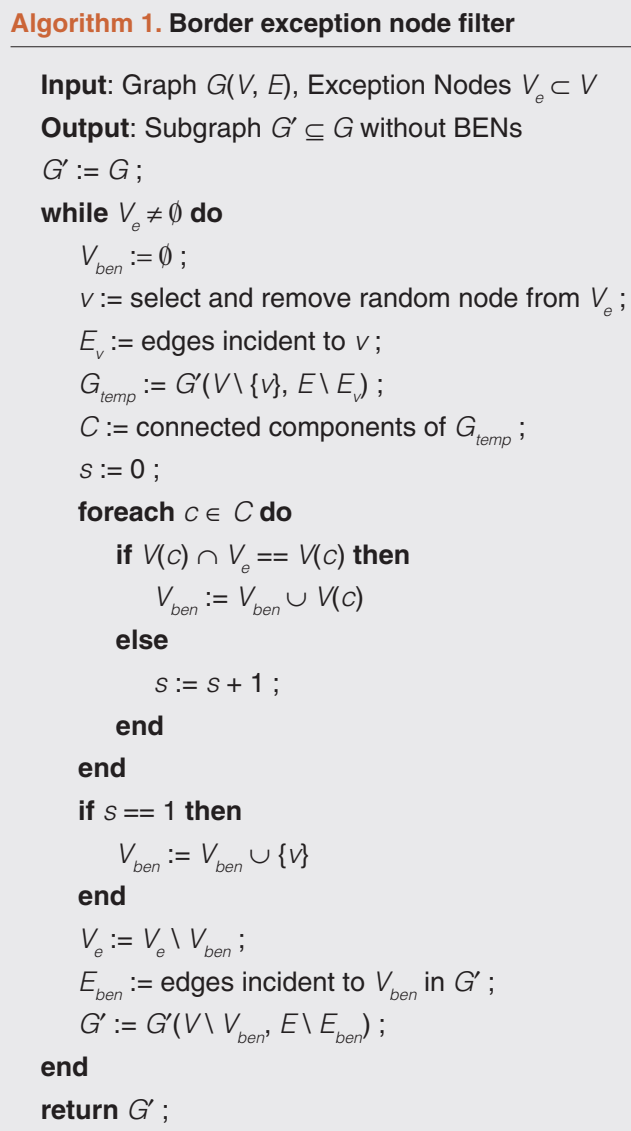

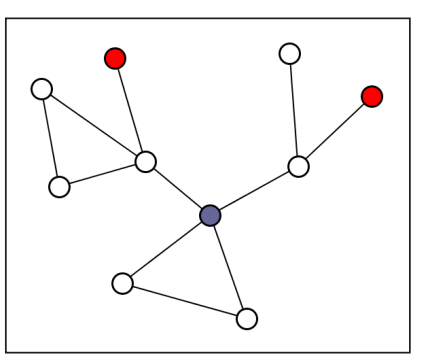
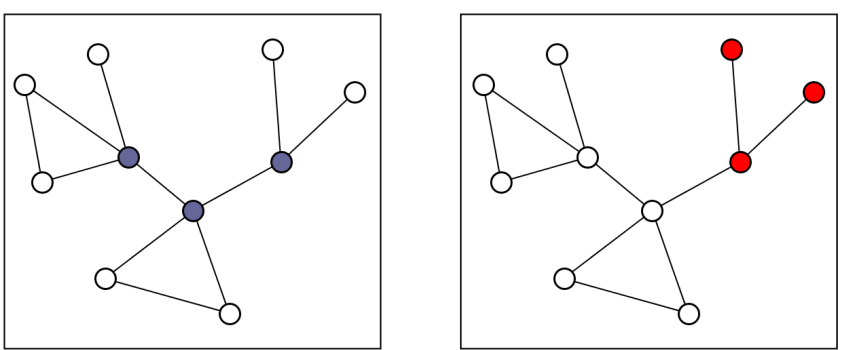

Figure 2. Three putative examples of solutions obtained with $K=3$ exception nodes to illustrate the impact of border exception nodes (BENs). Removing BENs (red) would not disconnect regions containing no exception nodes (white). In contrast, removing non-BEN exception nodes (dark grey) would create two disjoint subnetworks containing non-exception nodes. 


\section{Use cases}

We tested the usability of the new KeyPathwayMiner features with a gene expression dataset consisting of tissue samples from the caudate nucleus region of the brain ${ }^{18}$ taken from 38 patients suffering from Huntington's disease (HD) and from 32 healthy patients in the control group. While it is known that huntingtin protein plays a major role in the development of the disease, the corresponding gene is not differentially expressed in approximately $40 \%$ of the patients. Hence, it will not be found in an analysis focused on identifying differentially expressed genes. However, it can be expected that protein-protein interaction (PPI) partners of the huntingtin protein are differentially expressed on the transcript level, thus posing an ideal test case for KeyPathwayMiner, which can identify subnetworks with huntingtin as an exception node. The Human Protein Reference Database (HPRD version 9, http://www.hprd.org/download) ${ }^{19}$ was used as the interaction network. To produce an indicator matrix for down-regulation, a one-tailed t-test was used to compute p-values for each gene and patient in the disease group vs all patients in the control group. Afterwards, a p-value cutoff of 0.05 was selected to set a 1 (significant) or 0 (not significant) in the indicator matrix for downregulation (file available at http://keypathwayminer.compbio.sdu. dk/downloads/matrix-hd-down.dat).

In a first use case we performed a robustness analysis with KeyPathwayMiner (INES model, greedy algorithm) by fixing parameters to $L=20 \%$ ( 8 out of 32 ) of the cases and $K=5$ exception nodes. In other words, we searched for maximal connected subgraphs containing at most 5 nodes with no more than $20 \%$ of cases in which the gene represented by the network node is not down-regulated.

In a typical robustness scenario, we wanted to test how the solutions change when a certain percentage of the edges in the graph are removed randomly. We thus selected "edge removal" as the perturbation technique with perturbation levels selected to range from $10 \%$ to $50 \%$ in increments of $10 \%$. For each perturbation level, we created 10 randomly perturbed networks and executed KeyPathway Miner with identical settings as in the original run.

As one would expect, removing a certain percentage of edges reduced the overlap with the results from the original network. However, even after removing $50 \%$ of the edges (Figure 3), a moderate Jaccard index overlap of 0.45 was observed.

In a second use case, we give an example of a validation run. To this end, we compiled a gold standard gene set consisting of HD relevant genes (file available at http://keypathwayminer.compbio. sdu.dk/downloads/htt-relevant.txt) from the $\mathrm{KEGG}^{20,21} \mathrm{HD}$ and calcium signaling pathways. Calcium signaling has been suggested to have an important role in the development of $\mathrm{HD}^{22}$.

In this scenario we wanted to see how solutions overlap with gold standard gene sets when randomly shuffling the node labels. In addition to the indicator matrix for down-regulation, we also aimed at finding solutions containing up-regulated genes. Hence we produced an additional indicator matrix for up-regulation (file available at http://keypathwayminer.compbio.sdu.dk/downloads/matrix-hd-up.dat) and connected them both with an 'OR' operator. We set a common $L=15 \%$ for both sets together with $K=5$. KeyPathwayMiner (INES model, greedy algorithm) thus searched for pathways containing at most five genes with at most $L=15 \%$ genes that are not differentially regulated. The perturbation technique chosen was "node label permutation". The results show that even when permuting $80 \%$ of the node labels the overlap with the gold standard set remains relatively stable. As expected, we can see a significant drop when all labels are permuted (Figure 4).

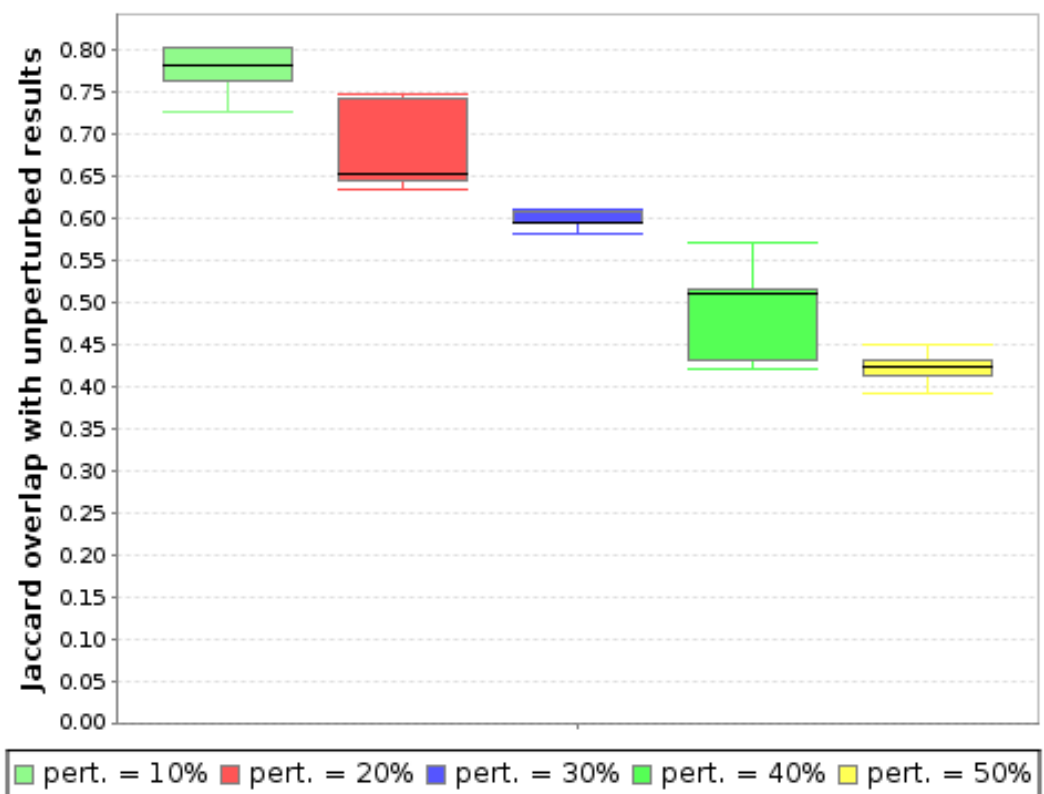

Figure 3. Robustness results for different percentages of edge removal. For each perturbation level, 10 different networks were generated randomly and submitted to KeyPathwayMiner for analysis. (Parameters: INES, greedy, $K=5, L=20 \%$ ) 


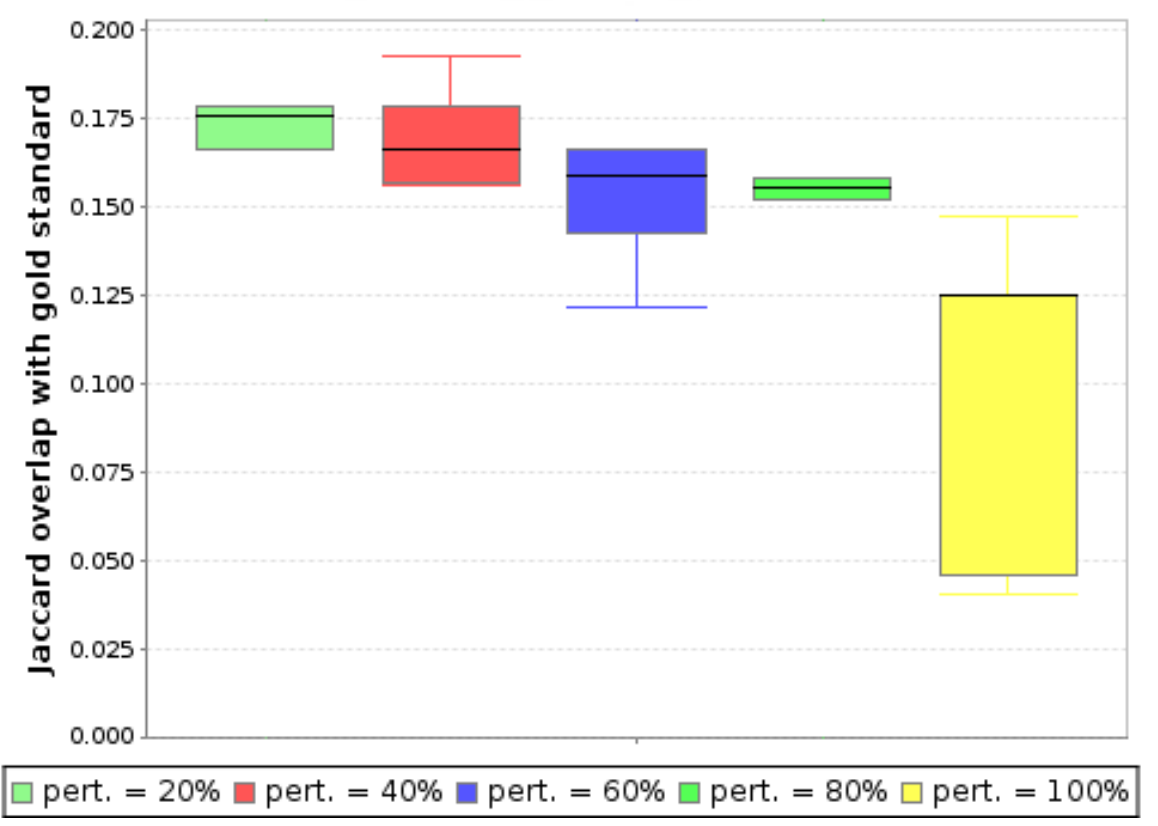

Figure 4. Overlap with the selected HD gold standard gene set with different percentages of permuted node labels in the input network. For each perturbation degree, 10 graphs were generated. (Parameters: INES, greedy, $K=5, L=15 \%$ ). Note that partial network perturbation and subsequent comparison with gold standard sets has limited meaning. Biological interaction networks are scale-free, i.e. robust to random perturbations. Of major importance for this effect are a small number of hub nodes. KeyPathwayMiner is able to recover subnetworks containing relevant genes connected to such hubs unless the hubs themselves are affected by the perturbation. This, however, is only the case when $100 \%$ of the network is perturbed (a randomized, true null model), explaining the performance drop we observe for this degree of perturbation.

\section{Dataset 1. Use case data of de novo pathway enrichment with KeyPathwayMiner 5}

http://dx.doi.org/10.5256/f1000research

Data from use cases are provided. Please see text file for a description of each set of data.

\section{Summary}

De novo pathway enrichment is a powerful method for the analysis of one or several types of molecular profiles. In contrast to widely used gene set enrichment methods such as GSEA ${ }^{23}$, this methodology is not limited to existing knowledge but suitable to uncover new functional modules. Results are extracted using large biological interaction networks, which are incomplete and continuously evolve. It is typically unclear how future updates leading to an interaction network of higher quality will affect the currently obtained results. KeyPathwayMiner 5 enables users to study the robustness of their results by allowing them to introduce artificial noise into the underlying interaction networks. Moreover, an existing gold standard can be used to test how well the optimal solution can be recovered on these perturbed networks.

\section{Data availability}

F1000Research: Dataset 1. Use case data of de novo pathway enrichment with KeyPathwayMiner 5, 10.5256/f1000research. 9054.d12687124

\section{Software availability}

1. Software available from: http://apps.cytoscape.org/apps/ keypathwayminer

2. Latest source code: https://github.com/baumbachlab/ keypathwayminer-cytoscape3 https://github.com/baumbachlab/keypathwayminer-cytoscape3/archive/5.0.tar.gz (KeyPathwayMiner Cytoscape app source code) https://github.com/baumbachlab/keypathwayminer-core/ archive/5.0.tar.gz (KeyPathwayMiner core library source code)

3. Archived source code as at time of publication: Zenodo, Source codes de novo pathway enrichment with KeyPathwayMiner, doi: 10.5281/zenodo55734 ${ }^{25}$

4. License: GPL v3 


\section{Author contributions}

JB, MR, NA, and ML specified the new features and plots that were implemented by MDH and NA. NA, ML and JB wrote the manuscript. All authors contributed to testing the software and have read and approved the final version of the manuscript.

\section{Competing interests}

No competing interests were disclosed.

\section{Grant information}

This work was supported by the Lundbeckfonden grant for the NanoCAN Center of Excellence in Nanomedicine, the Region
Syddanmarks ph.d.-pulje and Forskningspulje, the Fonden Til Lægevidenskabens Fremme, by the DAWN-2020 project financed by Rektorspuljen SDU2020 program, the MIO project of the OUH Frontlinjepuljen, the Bioinformatics part of NEXT - National Experimental Therapy Partnership funded by the Innovation Fund Denmark, as well as the VILLUM foundation by a Blokstipendiet. NA would like to acknowledge el Consejo Nacional de Ciencia y Tecnología (CONACyT) from Mexico for their financial support.

The funders had no role in study design, data collection and analysis, decision to publish, or preparation of the manuscript.
1. Chatr-Aryamontri A, Breitkreutz BJ, Oughtred R, et al:: The BioGRID interaction database: 2015 update. Nucleic Acids Res. 2015; 43(Database issue): D470-8. PubMed Abstract | Publisher Full Text | Free Full Text

2. Orchard S, Ammari M, Aranda B, et al:: The MIntAct project--IntAct as a common curation platform for 11 molecular interaction databases. Nucleic Acids Res. 2014; 42(Database issue): D358-63.

PubMed Abstract | Publisher Full Text | Free Full Text

3. Brown KR, Jurisica I: Unequal evolutionary conservation of human protein interactions in interologous networks. Genome Biol. 2007; 8(5): R95. PubMed Abstract | Publisher Full Text | Free Full Text

4. Beisser D, Brunkhorst S, Dandekar T, et al.: Robustness and accuracy of functional modules in integrated network analysis. Bioinformatics. 2012; 28(14): 1887-1894

PubMed Abstract | Publisher Full Text

5. Beisser D, Klau GW, Dandekar T, et al: BioNet: an R-Package for the functional analysis of biological networks. Bioinformatics. 2010; 26(8): 1129-30. PubMed Abstract | Publisher Full Text

6. Breitling R, Amtmann A, Herzyk P: Graph-based iterative Group Analysis enhances microarray interpretation. BMC Bioinformatics. 2004; 5: 100. PubMed Abstract | Publisher Full Text | Free Full Text

7. Nacu S, Critchley-Thorne R, Lee $\mathrm{P}$, et al:: Gene expression network analysis and applications to immunology. Bioinformatics. 2007; 23(7): 850-858. PubMed Abstract | Publisher Full Text

8. Vandin F, Upfal E, Raphael BJ: Algorithms for detecting significantly mutated pathways in cancer. J Comput Biol. 2011; 18(3): 507-22. PubMed Abstract | Publisher Full Text

9. Ideker T, Ozier O, Schwikowski B, et al.: Discovering regulatory and signalling circuits in molecular interaction networks. Bioinformatics. 2002; 18(Suppl 1): S233-S240.

PubMed Abstract | Publisher Full Text

10. Ulitsky I, Shamir R: Identification of functional modules using network topology and high-throughput data. BMC Syst Biol. 2007; 1: 8 . PubMed Abstract | Publisher Full Text | Free Full Text

11. Chuang HY, Lee E, Liu YT, et al:: Network-based classification of breast cance metastasis. Mol Syst Biol. 2007; 3: 140 PubMed Abstract | Publisher Full Text | Free Full Text

12. Qiu $Y Q$, Zhang S, Zhang XS, et al.: Detecting disease associated modules and prioritizing active genes based on high throughput data. BMC Bioinformatics. 2010; 11: 26

PubMed Abstract | Publisher Full Text | Free Full Text

13. List M, Alcaraz N, Dissing-Hansen M, et al.: KeyPathwayMinerWeb: online multiomics network enrichment. Nucleic Acids Res. 2016; pii: gkw373.

PubMed Abstract | Publisher Full Text
14. Alcaraz N, Pauling J, Batra R, et al.: KeyPathwayMiner 4.0: condition-specific pathway analysis by combining multiple omics studies and networks with Cytoscape. BMC Syst Biol. 2014; 8: 99 PubMed Abstract | Publisher Full Text | Free Full Text

15. Alcaraz N, Kücük H, Weile J, et al.: KeyPathwayMiner: Detecting Case-Specific Biological Pathways Using Expression Data. Internet Mathematics. 2011; 7(4): 299-313. Publisher Full Text

16. Alcaraz $\mathrm{N}$, Friedrich $\mathrm{T}$, Kötzing $\mathrm{T}$, et al:: Efficient key pathway mining: combining networks and OMICS data. Integr Biol (Camb). 2012; 4(7): 756-64. PubMed Abstract | Publisher Full Text

17. Maslov S, Sneppen K: Specificity and stability in topology of protein networks. Science. 2002; 296(5569): 910-913. PubMed Abstract | Publisher Full Text

18. Hodges A, Strand AD, Aragaki AK, et al.: Regional and cellular gene expression changes in human Huntington's disease brain. Hum Mol Genet. 2006; 15(6): 965-977.

PubMed Abstract | Publisher Full Text

19. Prasad TS, Kandasamy K, Pandey A: Human Protein Reference Database and Human Proteinpedia as discovery tools for systems biology. Methods Mol Biol. 2009; 577: 67-79. PubMed Abstract | Publisher Full Text

20. Kanehisa M, Goto S: KEGG: kyoto encyclopedia of genes and genomes. Nucleic Acids Res. 2000; 28(1): 27-30. PubMed Abstract | Publisher Full Text | Free Full Text

21. Kanehisa $M$, Sato $Y$, Kawashima $M$, et al.: Kegg as a reference resource for gene and protein annotation. Nucleic Acids Res. 2016; 44(D1): D457-D462. PubMed Abstract | Publisher Full Text | Free Full Text

22. Rockabrand E, Slepko N, Pantalone A, et al:: The first $\mathbf{1 7}$ amino acids of Huntingtin modulate its sub-cellular localization, aggregation and effects on calcium homeostasis. Hum Mol Genet. 2007; 16(1): 61-77. PubMed Abstract | Publisher Full Text

23. Subramanian A, Tamayo $P$, Mootha VK, et al:: Gene set enrichment analysis: a knowledge-based approach for interpreting genome-wide expression profiles. Proc Natl Acad Sci U S A. 2005; 102(43): 15545-50. PubMed Abstract | Publisher Full Text | Free Full Text

24. Alcaraz N, List M, Dissing-Hansen M, et al:: Dataset 1 in: Robust de novo pathway enrichment with KeyPathwayMiner 5. F1000Research. 2016. Data Source

25. Alcaraz N, List M, Dissing-Hansen M, et al:: Source codes de novo pathway enrichment with KeyPathwayMiner. Zenodo. Data Source 


\section{Open Peer Review}

\section{Current Peer Review Status:}

\section{Version 1}

Reviewer Report 28 July 2016

https://doi.org/10.5256/f1000research.9744.r15263

(C) 2016 Riemenschneider M. This is an open access peer review report distributed under the terms of the Creative Commons Attribution License, which permits unrestricted use, distribution, and reproduction in any medium, provided the original work is properly cited.

\section{Mona Riemenschneider}

Department of Bioinformatics, Straubing Center of Science, Weihenstephan-Triesdorf University of Applied Science, Straubing, Germany

The manuscript describes a tool for robust de novo pathway enrichment. The software provides the great advantage to study effects of network perturbations thereby allow for the evaluation of quality and robustness of the results in de novo pathway enrichment. Thus, the authors address a relevant issue in network construction and pathway enrichment.

The rationale for the development of the tool is clearly stated. A use case to demonstrate the usability of KeyPathwayMiner 5 with varying parameters is described within the manuscript. The source code of KeyPathwayMiner 5 is available at github.com.

\section{MINOR COMMENTS:}

The introduced tool KeyPathwayMiner 5 is a further development of KeyPathwayMiner x. A short overview of added features to all updated versions may be helpful for users to get an overview of the full function of KeyPathwayMiner 5. (provide in supplement)

Several parameters must be set to run KeyPathwayMiner. Could an approximate recommendation for parameter values and settings be given for non-expert users?

The calculation of the Jaccard index is given in detail, however a short explanation of the graduation (high, moderate, low) of calculated values could be helpful to provide easy interpretation of results for all users.

Please check spelling throughout the manuscript: KeyPathwayMiner/KeyPathway Miner

Competing Interests: No competing interests were disclosed. 
I confirm that I have read this submission and believe that I have an appropriate level of expertise to confirm that it is of an acceptable scientific standard.

Reviewer Report 01 July 2016

https://doi.org/10.5256/f1000research.9744.r14664

(C) 2016 Calderone A. This is an open access peer review report distributed under the terms of the Creative Commons Attribution License, which permits unrestricted use, distribution, and reproduction in any medium, provided the original work is properly cited.

\begin{abstract}
Alberto Calderone
Bioinformatics and Computational Biology Unit, Molecular Genetics Laboratory - Tor Vergata University, Rome, Italy

The article presents an update to a previously published Cytoscape App by providing a general overview of other methods. The title, as well as the abstract give a good introduction to the article.

The authors give a good recap of other methods and possible approaches as well as presenting the updated app. The Cytoscape app presentation is clear and enough for the final user. Most importantly, the new feature introduced in V5 - i.e. Perturbation - is clearly described in a dedicated paragraph. "Robust" in the title is justified in the text and use cases were clear and doable. Border exception node is illustrated clearly as well.

I could reproduce the examples given with no big problem. Overall, the article is well written.

Competing Interests: No competing interests were disclosed.

I confirm that I have read this submission and believe that I have an appropriate level of expertise to confirm that it is of an acceptable scientific standard.
\end{abstract}


The benefits of publishing with F1000Research:

- Your article is published within days, with no editorial bias

- You can publish traditional articles, null/negative results, case reports, data notes and more

- The peer review process is transparent and collaborative

- Your article is indexed in PubMed after passing peer review

- Dedicated customer support at every stage

For pre-submission enquiries, contact research@f1000.com 